\title{
Inhalt, Vol. 12, No. 1, 1989
}

\section{Contents}

\section{Impressum}

Hiddemann, W. (Münster) et al.*

Altersbezogener randomisierter Vergleich von sequentiell applizierten hochdosiertem versus intermediär dosiertem CytosinArabinosid in Kombination mit Mitoxantron (S-HAM) in der Therapie rezidivierter und refraktärer akuter myeloischer Leukämien: Studienkonzept und vorläufige Ergebnisse

Linkesch, W. (Wien/Österreich) et al.* Amsacrine, Cytarabin und Thioguanin (AAT) versus Daunoru-bicin, Cytarabin, Thioguanin (DAT) bei Erwachsenen mit unbe-handelter akuter nicht-lymphatischer Leukämie (ANLL): Ergebnisse aus Österreieh und der Bundesrepublik Deutschland . . Informationen für die Klinik

12

Gamer, A.; Ottmann, G.; Hoelzer, A. (Frankfurt), Schulz, G. (Marburg)* Therapie von Patienten mit myelodysplastischen Syndromen mit Granulozyten-Makrophagen-Kolonie-stirnulierendem Faktor (rhGM-CSF) und Cytosin-Arabinosid 13 Gattringer, C.; Thaler, J.; Orach, J.; Huber, H. (Innsbruck/ Österreieh), Micksche, M. (Traiskirchen/Österreich)* GM-CSF Behandlung in der Aplasie nach zytostatischen Therapien 16

Steinke, B. (Tubingen) et al.*

Sequentiell alternierende Chemotherapie bei hochmalignen Non-Hodgkin-Lymphomen mit VIM-Bleo und CHOP. Erste Ergebnisse 19

Gerhartz, H.H. (München) et al.*

Risiko-adaptierte Therapie hochmaligner Non-Hodgkin-

Lymphome mit COP-BLAM/IMVP-16: Eine prospektive multizentrische Studie

22

Preis, P. (Wien/Österreich) et al.* VMCP-Chemotherapie mit oder ohne Interferon-alpha-2 bei neudiagnostizierten Patienten mit multiplem Myelom

Imprint

Hiddemann, W. (Münster) et al.* 
Age Related Randomized Comparison of Sequentially Applied High-Dose versus Intermediate Dose Cytosine Arabinoside in Combination with Mitoxantrone (S-HAM) in the Treatment of Relapsed and Refractory Acute Myeloid Leukemia: Study Design and Preliminary Results

Linkesch, W. (Vienna/Austria) et al.*

Amsacrine, Cytarabine and Thioguanine (AAT) versus

Daunorubicin, Cytarabine, Thioguanine (DAT) in Adults with Untreated Acute Non-Lymphoblastic Leukemia (ANLL). Aus trian and German Results

12

Clinical Information

Ottmann, G.; Hoelzer, A. (Frankfurt), Schulz, G.

Ganser, A.. (Marburg)*

Recombinant Human Granulocyte-Macrophage Colony-

Stimulating Factor and Low-Dose Cytosine Arabinoside in

Patients with Myelodysplastic Syndrome 13

Gattringer, C; Thaler, J.; Drach, J.; Huber, H. (Innsbruck/

Austria), Micksche, M. (Traiskirchen/Austria)*

GM-CSF Treatment in Aplasia after Cytotoxic Therapy 16

Steinke, B. (Tubingen) et al.*

Sequential Alternating Chemotherapy in High-Grade Malignant Non-Hodgkin's Lymphomas with VIM-Bleo and CHOP. Pre liminary Results 19

Gerhartz, H.H. (Munich) et al.*

Risk-Adapted Therapy of Aggressive Non-Hodgkin's Lym phomas with COP-BLAM/IMVP-16: A Prospective Multicenter Trial 22

Preis, P. (Vienna/Austria) et al.*

VMCP-Chemotherapy With or Without Interferon-alpha-2 in

Newly Diagnosed Patients With Multiple Myeloma 27

Sonderbände

29

Special Editions.

29

33

Hartensteín, R.C.; Wendt, T.G.; Kastenbauer, E.R. (München), Trott, K.R. (London/GB)* Simultane Chemo-Radiotherapie mit 5-Fluorouracil/Folinsäure/ Cisplatin und hyperfraktionierter Bestrahlung bei fortgeschrittenen HNO-Karzinomen 30

Hinweise für Autoren.

Buchbesprechungen 35

Voigtmann, R. (Bochum) 
Knochenmarksnekrose bei einer Patientin mit metastasierendem Mammakarzinom unter Chemotherapie mit Chlorambucil, Methotrexat und Prednison 36

Kurzmitteilungen

Karstens, J.H.; Schnabel, B.; Ammon, J. (Aachen)

Behandlung von metastasenbedingtem Knochenschmerz: erste

Ergebnisse einer Studie mit Einzelfraktions-(4 Gy) Strahlen-

therapie $\quad 41$

Sommerkamp, H. (Freiburg)

Eingeschränkte Therapie beim Prostatakarzinom 44

* «Aktuelle Therapiestudien» - Referate gehalten anläßlich dcr Jahrestagung dcr

Österrcichischen und Deutschen Gesellschaften für Hämatologie und Onkologie, Salzburg,

Oktober 1988

Hartenstein, R.C.; Wendt, T.G.;

3335

Kastenbauer, E.R. (Munich), Trott, K.R. (London/GB)*

Simultaneous Chemo-Radiotherapy with 5-FU/Folinic/Acid/cis-

Platinum and Accelerated Split-Course Radiation in Advanced

Head and Neck Cancer 30

Instructions to Authors Book Reviews ....

Voigtmann, R. (Bochum)

Bone Marrow Necrosis in a Patient With Metastatic Breast

Cancer Receiving a Cytostatic Therapy With Chlorambucil,

Methotrexate and Prednisone. A Case Report 36

Short Communications

Karstens, J.H.; Schnabel, B.; Ammon, J. (Aachen)

Management of Metastatic Bone Pain: Preliminary Results With

Single Fraction (4 Gy) Radiotherapy 41

Sommerkamp, H. (Freiburg)

Limited Therapy of Prostatic Carcinoma

44

* "Current Therapy Studies" - presented at the Joint Annual Meeting of the German and Austrian Societies of Hcmatology and Oncology, Salzburg, October 1988

Bibliographischer Hinweis: Inhaltsverzeichnisse dieser Zeitschrift erscheinen regelmäßig in current contents ${ }^{\circledR}$ sowie in anderen bibliographischen Diensten. 